\title{
Emulation of end-to-end communications systems in railway scenarios: physical layer results
}

Garcia-Loygorri, Juan Moreno; Kharbech, Sofiane; Clavier, Laurent; Kassi, Redha; Torrego, Raul; Arriola, Aitor; Val, Inaki; Berbineau, Marion; Soler, José; Yan, Ying

\section{Published in:}

Proceedings of $14<$ sup $>$ th $</$ sup $>$ European Conference on Antennas and Propagation

Link to article, DOI:

10.23919/EuCAP48036.2020.9135760

Publication date:

2020

Document Version

Peer reviewed version

Link back to DTU Orbit

\section{Citation (APA):}

Garcia-Loygorri, J. M., Kharbech, S., Clavier, L., Kassi, R., Torrego, R., Arriola, A., Val, I., Berbineau, M., Soler, J., \& Yan, Y. (2020). Emulation of end-to-end communications systems in railway scenarios: physical layer results. In Proceedings of 14 European Conference on Antennas and Propagation IEEE.

https://doi.org/10.23919/EuCAP48036.2020.9135760

\section{General rights}

Copyright and moral rights for the publications made accessible in the public portal are retained by the authors and/or other copyright owners and it is a condition of accessing publications that users recognise and abide by the legal requirements associated with these rights.

- Users may download and print one copy of any publication from the public portal for the purpose of private study or research.

- You may not further distribute the material or use it for any profit-making activity or commercial gain

- You may freely distribute the URL identifying the publication in the public portal 


\title{
Emulation of end-to-end communications systems in railway scenarios: physical layer results
}

\author{
Juan Moreno García-Loygorri ${ }^{1}$, Sofiane Kharbech ${ }^{2}$, Laurent Clavier ${ }^{2}$, Redha Kassi ${ }^{2}$, Raúl Torrego ${ }^{3}$, Aitor Arriola ${ }^{3}$, \\ Iñaki $\mathrm{Val}^{3}$,Marion Berbineau ${ }^{4}$, José Soler ${ }^{5}$, Ying Yan $^{5}$ \\ ${ }^{1}$ Área de Ingeniería, Metro de Madrid S.A., Spain, juan.moreno@metromadrid.es \\ ${ }^{2}$ Univ. Lille, CNRS, UMR 8520 - IEMN, F-59000 Lille, France \\ ${ }^{3}$ Communication Systems Group, Ikerlan Technology Research Centre, 20500 Arrasate-Mondragón, Spain \\ ${ }^{4}$ IFSTTAR, COSYS, LEOST, Villeneuve d'Ascq, F-59650 \\ ${ }^{5}$ DTU Fotonik, 2800 Kgs. Lyngby, Denmark
}

\begin{abstract}
The complexity of modern communication systems is remarkable, and the efforts needed to put into service a new one are substantial as well. In some industrial sectors, circumstances are even harder. For example, in railways, the tests to be done are costly due to the integration in the rolling stock plus the need to have physical access to the railway tracks. Therefore, it is worth having a suitable emulator that considers many different radio-access technologies (RAT) in several railway scenarios (viaducts, tunnels, rural, hilly, etc.). Moreover, it should be able to do an end-to-end emulation, absolutely transparent for the application layer (this is, considering not only the physical layer but the network one as well). In this paper, we highlight the physical layer aspects considered in the construction of this emulator. Integration with the network layer is briefly mentioned, as well as the whole architecture.
\end{abstract}

Index Terms - channel models, emulation, railways, vehicular.

\section{INTRODUCTION}

Train-to-ground (T2G) radio communication systems are a key building block in the ultra-reliable signaling systems that modern railways are today. A good example of these signaling systems is ERTMS (European Rail Traffic Management System) [1], which allows highspeed trains to achieve speeds up to $350 \mathrm{~km} / \mathrm{h}$ with 3 minutes' headway between trains. Another good example is CBTC (Communications-Based Train Control) [2] very similar to ERTMS but focused on subway trains, some of them completely automated with no staff on board.

The main problem with $\mathrm{T} 2 \mathrm{G}$ systems is the long timeto-market that these technologies have. To avoid a complicated validation process with costly on-site testing for new T2G, the European EMULRADIO4RAIL Project [3] will provide an innovative emulation platform for testing and validation of various radio access technologies (RAT), such as IEEE 802.11 (Wi-Fi), LTE, LTE-A and satellite communications. The emulation platform will combine simulations of the communication core network and emulation of various RATs, thanks to the coupling of discrete event simulators such as the
RIVERBED Modeler [4], Open Air Interface, several physical radio channel emulators, models of IP parameters and real physical systems.

In this paper, we will describe the overall architecture of the emulation platform, underlining the physical layer aspects that are considered in the project, as well as some of the very early results that we have. The organization of the paper is as follows: in Section II we describe the different railway scenarios and the perturbations that are emulated; in Section III the architecture of the whole emulator is presented; in Section IV the initial results of the emulation platform are outlined; and, finally, in Section $\mathrm{V}$ the main conclusions are provided.

\section{ENVIRONMENT}

\section{A. Railway scenarios}

The objective of this research work is to emulate as many railway-related scenarios as possible. The problem is that railways are very diverse, so, to address the complexity of railway environments, we have classified them into five different ones:

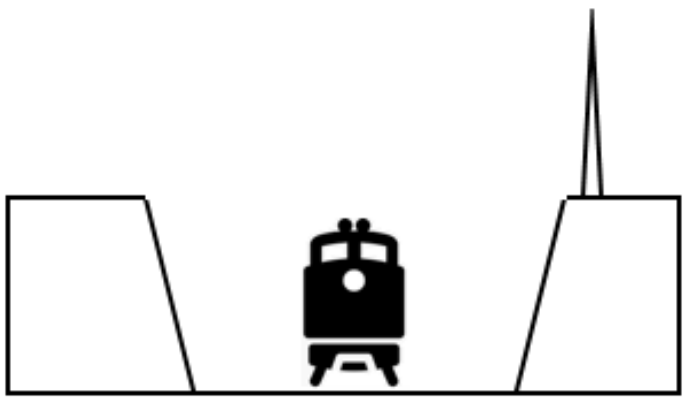

Fig. 1. Cutting scenario on a railway line.

- $\quad$ Rural: low building density, LOS assumed most of the time. It is usual in mainline, high-speed, and regional lines.

- Hilly terrain: usual scenario in railways around the world.

- Viaduct: very common in modern lines, in order to avoid steep slopes which could be unaffordable for high-speed trains. 
- Cutting: this scenario is very specific for modern highway and railway lines, in order to decrease the impact on neighboring areas (cf. Fig .1).

- Tunnel: commonplace in subways and often present in high-speed lines.

The idea behind this is that almost every railway line could be defined as a composite of all these five scenarios.

\section{B. Models}

For the sake of efficiency, it was agreed to perform the physical layer emulation using a tapped delay line (TDL) emulator. Moreover, this is the most used model in wideband channels. This model assumes that there are $\mathrm{K}$ taps, each of them with a delay and an associated complex coefficient, which could be easily emulated. One of the key aspects of our research is the need to have control over the Doppler Spectrum, which is very relevant in railway scenarios (train speed could be up to $350 \mathrm{~km} / \mathrm{h}$, probably higher in the medium-term).

In Fig.2, the general concept for this model can be seen, and the expression for the channel impulse response is:

$h(\tau, t)=\sum_{k=1}^{K} c_{k} \quad \delta\left(\tau-\tau_{k}\right)$

where $\tau$ is the delay and $t$ is time [5]. For more details on TDL lines please refer to [5].

$\mathrm{c}_{1}$

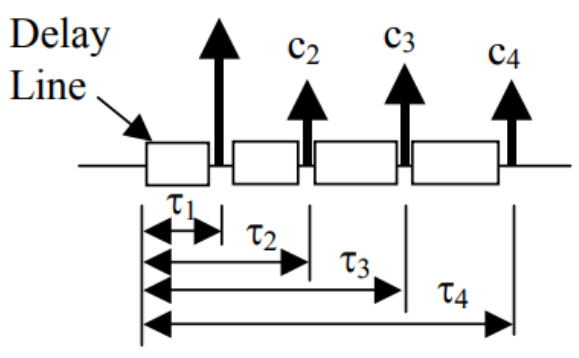

Fig. 2. Tapped Delay Line channel model

An exhaustive survey of the state-of-the-art radio channel models in railways has been done. The outcome is that in the literature, we can find many different TDLrelated channel models suitable for our purposes. We searched TDL-related channel models that contained the following information: number of taps, delay and relative power for each tap; speed range, Doppler spectrum frequency range, bandwidth, and diversity (MIMO, MISO, etc.). The major drawback is that, as far as we know, there are not TDL-based models for tunnels. This is still an open issue within the project which will be fixed as soon as possible. A very concise summary of the best models identified in this survey is shown in Table I. For more details on each one of them, please refer to the specific papers.

\section{Perturbations}

Perturbations, or electromagnetic interference (EMI), significantly impact the reliability of the wireless link. They include interference induced by other communication networks using the same or adjacent frequencies, transient EM interferences, produced by the catenary-pantograph contact loss (broadband and able to impact on the frequency bands of the different communication systems), Intentional EM Interferences (IEMI), produced by jammers onboard train or on the wayside. In many situations, EMI in a railway environment exhibits non-Gaussian statistics. Its dynamicity also has a strong impact. To consider interferences in both simulation and emulation, it is necessary to take into account that, depending on their origin, interferences do not have the same impact on the uplink and downlink. We can classify EM perturbations in three main categories:

- Network interference - coming from other devices communicating at the same time and frequency band. Densification of networks will certainly impact all types of railway communication. It should be avoided in licensed bands (cellular networks), adjacent cell interference is indeed present, and operators do not always take the high-speed specificities into account. Thus, out of band interference from adjacent (in frequency) networks may happen. The situation is more critical for unlicensed bands, especially if IEEE 802.11 (i.e., Wi-Fi) systems are used for critical communications (something common in urban railways). Especially dense environments, such as arrivals to train stations, are to be carefully studied.

- Unintentional jamming - it refers to equipment that does not have an EM radiating functionality, i.e., they are externalities. Many sources can produce such perturbations: high voltage overhead power lines, arcing and lightning, power converters and battery chargers, and other railway systems nearby [6]. They are generally impulsive with different rise times (slow pulses with high energy content, and fast pulses featuring much lower energy). The most significant source of impulsive disturbance, threatening reliability, is arcing produced by the sliding contact between the pantograph and the catenary or by the third rail $[7,8,9]$.

- Illegal jamming - current telecommunication solutions make it easy to access to EM signal generation equipment (i.e., Software-Defined Radio modules), which can be used for either legitimate or malicious purposes $[10,11]$. The existence of telecommunication jammers is widely noticed, and it constitutes a risk for a large number of communication services [12]. Many types of radio jammers are for sale on the market, batteryoperated, pocket-size, or more powerful. Some can block the signals of only one frequency band; some can block up to five frequency bands at the same time [13]. They operate from several hundred $\mathrm{MHz}$ up to a few $\mathrm{GHz}$. Most of the proposed solution uses a sweeping strategy where a transmitted sine wave with a changing frequency rapidly covers the frequency band. Their instantaneous frequency varies as a linear function of 


\begin{tabular}{|c|c|c|c|c|c|}
\multicolumn{2}{c|}{ Table I: summary of channel models to be emulated in the platform } \\
\hline \multirow{2}{*}{ Scenario } & Frequency & Speed & SISO/MIMO & $\begin{array}{c}\text { Doppler } \\
\text { spectrum } \\
\text { information } \\
\text { available }\end{array}$ & Reference \\
\hline Hilly & $900 \mathrm{MHz}$ & $100 \mathrm{~km} / \mathrm{h}$ & SISO & Yes & {$[14]$} \\
\hline Rural & $450 \mathrm{MHz}-6 \mathrm{GHz}$ & $350 \mathrm{~km} / \mathrm{h}$ & $\mathrm{MIMO}$ & Yes & {$[15]$} \\
\hline Viaduct & $2.35 \mathrm{GHz}$ & $240 \mathrm{~km} / \mathrm{h}$ & SISO & Yes & {$[16]$} \\
\hline Cutting & $950 \mathrm{MHz} \& 2.15 \mathrm{GHz}$ & $310 \mathrm{~km} / \mathrm{h}$ & SISO & Yes & {$[17]$} \\
\hline Tunnel & - & - & - & - & - \\
\hline
\end{tabular}

time. To emulate a radio link in a railway scenario, it is essential to include these different perturbations either by models at the baseband level (models which in general are not Gaussian) or at the RF level with the true interfering signals (jammers, network interference) or replicas of recorded events (natural EMI).

\section{EMULATOR ARCHITECTURE}

\section{A. General architecture}

The global EMULRADIO4RAIL platform is intended for partners that aim to test their IP level communication devices against a very realistic emulation platform in which radio bearers, RF channels, and backhaul/PLMN (Public Land Mobile Network) will be emulated in real-time. The platform will combine simulations of the communication core network and emulation of various radio access technologies. Besides, it will couple the discrete event simulator RIVERBED Modeler (formerly OPNET) with a network emulator based on Open Air Interface (OAI) and various radio channel emulators. A schematic view of possible integration of the Riverbed modeler, OAI, and real systems is given in Fig.3. As we described in Section II, specific railway channel models and perturbation models will be implemented in the radio channel emulator.

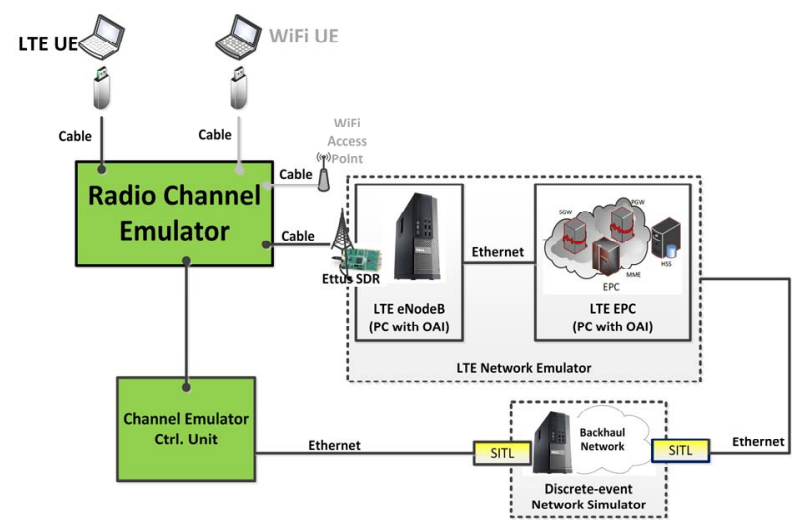

Fig. 3. Example of a possible combination of the Riverbed modeler, OAI, and real systems.

The project also develops an emulation platform for satellite links, as is explained in [18]. In this paper, we focus on the Wi-Fi and LTE radio access technologies (this is the channel emulators).

\section{B. Description of the channel emulators}

Two channel emulators are considered in this work. The first is based on reconfigurable hardware (FPGAs), the second one considers an existing commercial baseband generator and a built-in PXB channel emulator.

\section{1) The FPGA-based channel emulator}

The FPGA based channel emulator aims to provide the project with a fully customizable implementation (both at RF and baseband signal processing) of a channel emulator. The main features of this channel emulator are the following:

- Four bidirectional RF ports.

- $100 \mathrm{MHz}$ signal bandwidth.

- Tunable RF Frequency from $400 \mathrm{MHz}$ up to $6 \mathrm{GHz}$.

- FPGA implementation of the baseband signal processing algorithms (i.e., fading, SNR control, tap delay).

\section{2) The PXB-based baseband channel emulator}

The main features of the PXB N5106A baseband channel emulator, from Keysight Technologies, are the following:

- Input and output bandwidth:160 MHz (80 MHz I + 80 MHz Q).

- Many predefined channel models (GSM, 802.16, WLAN, etc.), including LTE communications for high-speed trains (vehicle speed up to $864 \mathrm{~km} / \mathrm{h}$ ).

- Supports MIMO systems (up to $4 \times 4$ ) and considers antenna correlation.

- Additive white Gaussian noise.

- Many fading distributions like Rice, Rayleigh, etc., and Doppler spectra (flat, Jakes, etc.) as well.

- Up to 24 taps per fader.

\section{EXPERIMENTAL VALIDATION.}

In this section, we present some preliminary results from both channel emulators. In the first one, the FPGAbased, we focus on some physical layer validation regarding the power-delay profile (PDP) and the correct emulation of the Doppler spectrum. In the second one, the PXB-based channel emulator, to determine the validity of the channel emulator for upper layers, we provide results in terms of bandwidth, jitter, and packet loss.

\section{1) FPGA-based channel emulator}

At the current state of the project, a set of measurements has been made to verify the correct operation of the channel emulator before connecting it to the final Devices-Under-Test (DUT). Fig. 4 shows the setup used for these measurements:

- A signal generator (E4438C by Agilent Technologies / Keysight) which sends a multitone signal.

- The channel emulator which modifies this signal, adding all the considered channel effects. 
- A Vector Signal Analyzer (VSA 89600 by Agilent Technologies / Keysight) that samples the modified signal.

- A PC that post-processes the digitized signal in order to obtain the channel impulse response.

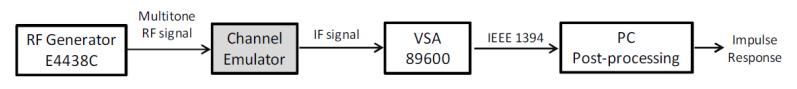

Fig. 4. Validation setup.

To check the multipath emulation capabilities of the channel emulator, several channels with different attenuation and delay values for each path have been tested. The PDP has been measured for each case. For example, Fig. 5 shows the PDP of a two-tap channel with the second path at $-6 \mathrm{~dB}$ and $320 \mathrm{~ns}$ from the first one $(0$ $\mathrm{dB}, \mathrm{t}=0 \mathrm{~ns})$. In Fig. 5, both taps can be seen, and the fitting to the expected result is remarkable.

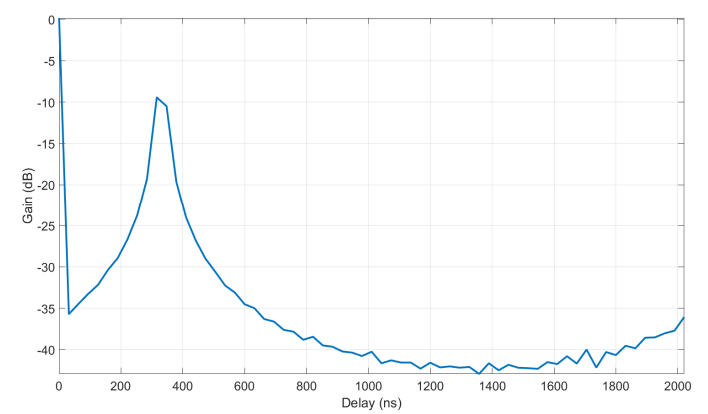

Fig. 5. Power delay profile of a two-tap channel. $1^{\text {st }}$ tap is at $0 \mathrm{~dB}$ and $0 \mathrm{~ns} ; 2^{\text {nd }}$ tap is expected $6 \mathrm{~dB}$ down and $320 \mathrm{~ns}$ delayed from the first one.

Regarding the validity of the fading generator implemented in the emulator, different Doppler power spectra have been programmed and measured as well. In Fig. 6, an example shows the measured power spectral density of a one-tap channel with a $20 \mathrm{~Hz}$ Jakes' fading. The adjustment to the theoretical shape is noteworthy.

Fig. 6. Power spectral density for a 1 tap, $20 \mathrm{~Hz}$ Jakes doppler.

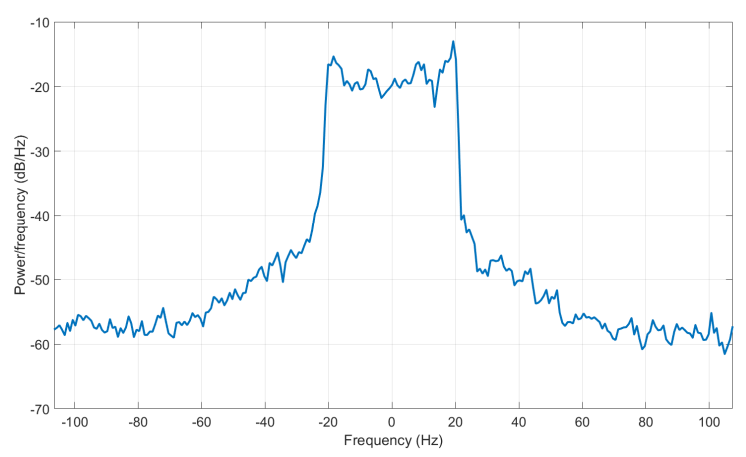

2) $P X B$ channel emulator

The experiments aim at emulating a radio channel for the downlink of an IEEE 802.11g end-to-end communication. Figure 7 shows the related testbed.

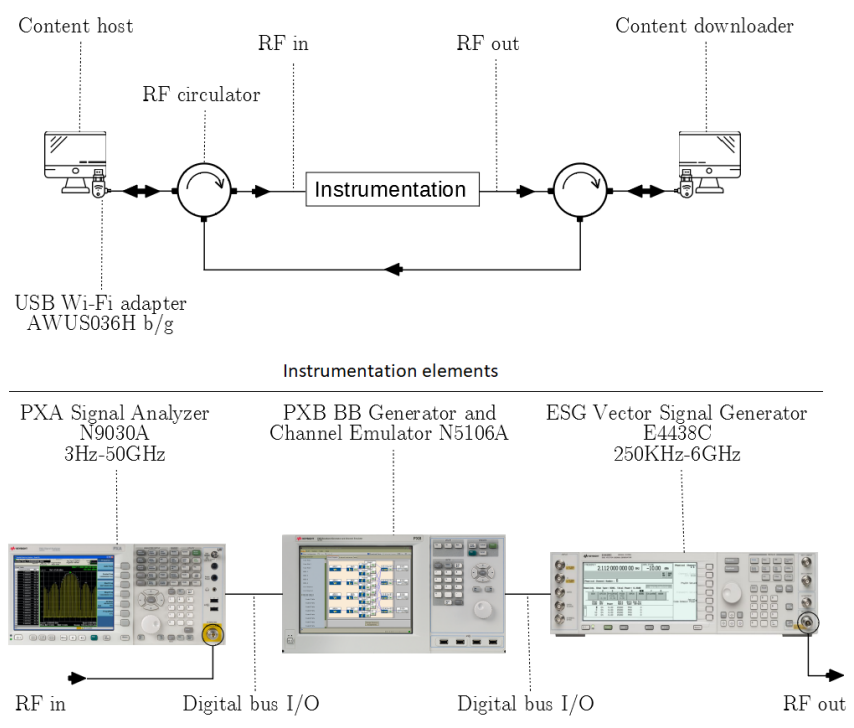

Fig. 7. PXB-based emulation testbed.

Tests were done using the N5106A PXB baseband generator and channel emulator from Keysight. Since it is a baseband emulator, RF signals need to be downconverted (PXA) before passing through the PXB and then up-converted (ESG) at its output.

For these initial tests that are covered here, the emulated channel is one of the preconfigured channels in the PXB; an 18-taps-TDL Rayleigh fading with $250 \mathrm{~ns}$ average RMS delay spread. End-to-end communication operates on a frequency of $2.412 \mathrm{GHz}$. Channel's time variation is modeled by the classical $6 \mathrm{~dB}$ U-shaped Doppler spectrum. The testing scenario consists of transmitting iPerf traffic from the transmitter host to the receiver one, then measuring some of network QoS metrics. Test trials are performed through several batches of traffic for different vehicular velocities. Figures 8 and 9 show that faster channel time-variation caused by high vehicular speed is worsening end to end transmission performance.

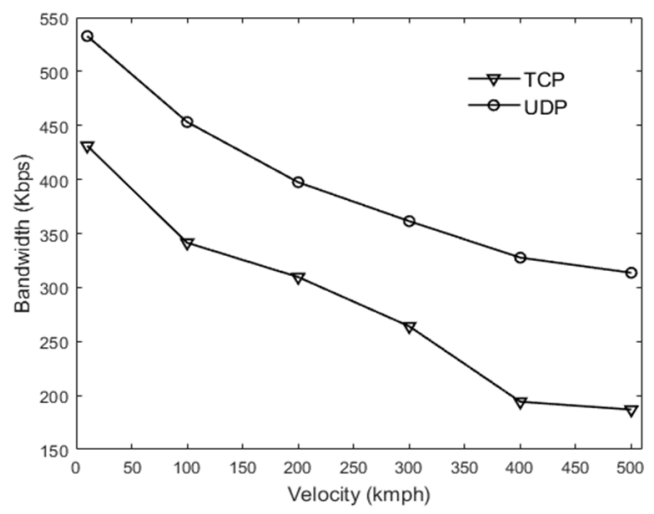

Fig. 8. Communication bandwidth in terms of velocity. The emulated channel is a 18-tap Rayleigh-fading with Jakes' Doppler spectrum at $2.4 \mathrm{GHz}$ using the PXB channel emulator. 


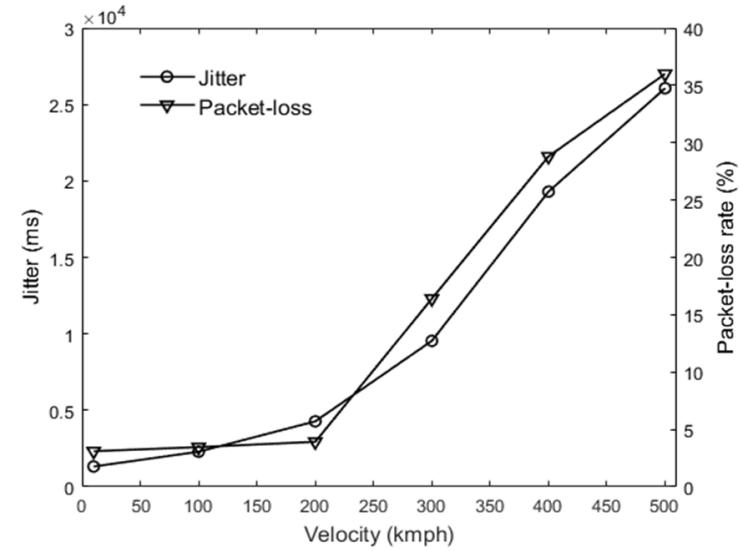

Fig. 9. Network jitter and packet-loss variations in terms of velocity. The emulated channel is a 18-tap Rayleigh-fading with Jakes' Doppler spectrum at $2.4 \mathrm{GHz}$ using the PXB channel emulator.

In Fig.8, both the TCP and UDP bandwidths for several different vehicular speeds are shown. In Fig. 9, both the packet-loss and the end-to-end jitter are severely worsened by the speed of the train. All these results are very preliminary but also promising because they are coherent with the expected outcome of emulation like this.

\section{CONCLUSIONS}

A platform able to perform an end-to-end emulation is of much interest for the validation of radio communication systems in railways, which now need many costly field tests. This platform must include a channel emulator able to take into account physical layer effects on the signal due to the channel itself (multipath, Doppler, etc.) and perturbations as well. This paper described the whole architecture of the emulation platform, with particular emphasis on the physical layer aspects, with some results of the validation.

\section{ACKNOWLEDGMENT}

This work is performed in the framework of the Emulradio4rail project that has received funding from the European Union's Horizon 2020 research and innovation program under grant agreement No 826152.

\section{REFERENCES}

[1] J. Moreno, et. al, "A survey on the future railway radio communications services: challenges and opportunities", IEEE Communications Magazine, October 2015, pp. 62-68.

[2] M.Fitzmaurice, "Wayside Communications: CBTC Data Communications Subsystems", IEEE Vehicular Technology Magazine, September 2013, Volume: 8, Issue: 3, pp. 73-80.

[3] EmulRadio4Rail Project. [Online available]: http://www.emulradio4rail.eu

[4] Riverbed Modeler. [Online available]: https://cmsapi.riverbed.com/portal/community home

[5] A. F. Molisch, Wireless Communications: John Wiley \& Sons, Ltd, 2011.

[6] A. Ogunsola and A. Mariscotti, Electromagnetic Compatibility in Railways: Analysis and Management, Springer Science \& Business Media, 2012.
[7] S. Dudoyer, et. al, "Study of the susceptibility of the GSM-R communications face to the electromagnetic interferences of the rail environment," IEEE Transactions on electromagnetic compatibility, vol. 54, no. 3, pp. 667-676, Jun. 2012.

[8] A. Morant, et. al, "Railway EMI impact on train operation and environment", in the International Symposium on Electromagnetic Compatibility-EMC EUROPE, Sept. 2012, pp. 1-7.

[9] K. Hassan, et. al, "Multiple-Antenna-Based Blind Spectrum Sensing in the Presence of Impulsive Noise," in IEEE Transactions on Vehicular Technology, vol. 63, no. 5, pp. 22482257, Jun 2014.

[10] V. Deniau, et. al, "IEEE 802.11n communications in the presence of frequency-sweeping interference signals," IEEE Transactions on Electromagnetic Compatibility, vol. PP, no. 99, pp. 1-9, 2017.

[11] C. Kasmi and J. L. Esteves, "IEMI threats for information security: Remote command injection on modern smartphones," IEEE Trans. Electromagn. Compat., vol. 57, no. 6, pp. 17521755, Dec. 2015

[12] R. R. Tanuhardja, et. al, "Vulnerability of terrestrial-trunked radio to intelligent intentional electromagnetic interference," IEEE Trans. Electromagn. Compat., vol. 57, no. 3, pp. 454-460, Jun. 2015

[13] M. Heddebaut, et. al, "Mitigation techniques to reduce the vulnerability of railway signaling to radiated intentional EMI emitted from a train," IEEE Transactions on Electromagnetic Compatibility, vol. 59, no. 3, pp. 845-852, 2017.

[14] ETSI 145005 V13.3.0 Digital cellular telecommunications system (Phase 2+) GSM: GSM-EDGE Radio transmission and reception. 3GPP TS $45.0145 \quad 005 \quad$ V13.3.0 Release 13 https://www.etsi.org/deliver/etsi ts/145000 145099/145005/13. 03.00_60/ts_145005v130300p.pdf

[15] M. Series, "Guidelines for evaluation of radio interface technologies for IMT-Advanced,” Report ITU, no. 2135-1, 2009

[16] L. Liu et al., "Position-Based Modeling for Wireless Channel on High-Speed Railway under a Viaduct at 2.35 GHz,’ IEEE Journal on Selected Areas in Communications, vol. 30, no. 4, pp. 834 845, May 2012.

[17] J. Ding, et. al, "Broadband Wireless Channel in Composite HighSpeed Railway Scenario: Measurements, Simulation, and Analysis," Wireless Communications and Mobile Computing, 2017.

[18] M. Berbineau, et. al, "Emulation of various radio access technologies for zero-on-site testing in the railway domain - The Emulradio4rail platforms", Accepted for publication at TRA2020, April 2020. 